\title{
Spatially resolved Hall effect measurement in a single semiconductor nanowire
}

\author{
Kristian Storm, Filip Halvardsson, Magnus Heurlin, David Lindgren, Anders Gustafsson, \\ Phillip M. Wu, Bo Monemar and Lars Samuelson
}

\section{Linköping University Post Print}

N.B.: When citing this work, cite the original article.

Original Publication:

Kristian Storm, Filip Halvardsson, Magnus Heurlin, David Lindgren, Anders Gustafsson, Phillip M. Wu, Bo Monemar and Lars Samuelson, Spatially resolved Hall effect measurement in a single semiconductor nanowire, 2012, Nature Nanotechnology, (7), 11, 718-722. http://dx.doi.org/10.1038/nnano.2012.190

Copyright: Nature Publishing Group http://www.nature.com/

Postprint available at: Linköping University Electronic Press http://urn.kb.se/resolve?urn=urn:nbn:se:liu:diva-91718 


\title{
Hall effect measurement in a single semiconductor nanowire
}

\author{
Kristian Storm ${ }^{1}$, Filip Halvardsson, Magnus Heurlin, David Lindgren, Anders Gustafsson, \\ Phillip M. Wu, Bo Monemar and Lars Samuelson \\ Solid State Physics, the Nanometer Structure Consortium, Lund University \\ ${ }^{1}$ kristian.storm@ftf.lth.se
}

Establishing a sustainable global power consumption remains one of the greatest challenges of our time. Efficient light-emitting diodes (LEDs) and photo-voltaic (PV) energy harvesting is expected to play substantial roles in the continued development towards this goal. Semiconductor nanowires (NWs) have proven themselves a promising candidate for both $\mathrm{LED}^{1-6}$ and $\mathrm{PV}^{7-10}$ applications, but are yet to be fully implemented in production-grade devices. In order for the NW technology to make the transition to device-grade material and move past proof-of-concept devices, the ability to precisely quantify and control fundamental material properties such as doping and mobility is necessary. Unfortunately, some of the same properties that make NWs so interesting to study also make them inherently difficult to characterize. Here, we report a method to carry out Hall measurements on single core-shell NWs; enabling the spatially resolved conclusive determination of carrier concentration and mobility of the nanowire shell. Hall measurements have previously been completely unavailable for nanowire devices, and the measurement platform presented in this letter marks a leap forward in the capability to characterize and fabricate advanced engineered nanowire structures.

LED illumination has the potential to reduce the overall global electricity consumption by more than $10 \%^{11}$. However, one of the main challenges for energy efficient white LED illumination of optimal color-rendering index remains the so called green gap of the emission efficiency. In planar LED structures, this primarily stems from the difficulties involved in growing high In-content InGaN structures in which the high In content causes phase separation and strain-induced structural defects due to lattice parameter mismatch. Together with the electron-hole separation related to growth along the highly polarized c-plane this decreases the quantum efficiency. Nanowires promise to alleviate these problems ${ }^{4}$ by elastic strain relaxation due to high surface-to-volume ratio and the possibility to grow the active region along the non-polar m-plane. For PV devices, core-shell NW structures are predicted to allow large improvements in efficiency over the planar geometry by combining optimal absorption of photons with efficient collection of generated carriers. ${ }^{9}$

In order to optimize the performance of core-shell NW devices, it is predicted that advanced axial doping profile engineering must be carried out along the $\mathrm{NW}^{12}$. This requires methods to measure spatially resolved material properties along the NW axis. However, the size and geometry of NW devices have long made them elusive to most conventional electrical characterization techniques such as capacitance-voltage $(\mathrm{C}-\mathrm{V})$ spectroscopy and Hall characterization. 
Techniques that enable $\mathrm{C}-\mathrm{V}$ measurements on both single, laterally positioned, NWs as well as vertical NW arrays have recently been demonstrated ${ }^{13 ; 14}$, and in this paper we present a method to carry out Hall measurements on single core-shell NWs.

Figure 1a shows the device design under study in this work. Hexagonal InP p-type core, n-type shell zinc-blende NWs were grown by metal-organic vapor phase epitaxy on InP substrates. Each NW position was pre-determined using nano imprint lithography and the final as-grown structure can be seen in Fig. 1b. The InP NWs were transferred to an insulated Si substrate and a selfalignment technique was employed to deposit a polymer lifting layer. The lifting layer is designed to automatically align itself such that the top facet of the NW is exposed and made available for electrical characterization, see Fig. 1c and supplementary information Fig. 1 for details on the device design. The lifting layer eliminates the need for thick metal layers, thus enabling high spatial resolution, and furthermore allows NWs of different diameter to be characterized using the same process parameters.

Eight metal electrodes for sourcing currents and reading voltages were defined by electron beam lithography and deposited onto the NW shell by metal evaporation. By sourcing a current through terminals AH (see Fig. 1a) while simultaneously applying a magnetic field perpendicular to the sample surface, the Hall voltage across the NW shell can be measured between terminals BC, DE and FG denoted $V_{\mathrm{BC}}, V_{\mathrm{DE}}$ and $V_{\mathrm{FG}}$ respectively.

We first measured the Hall voltages $V_{\mathrm{BC}}, V_{\mathrm{DE}}$ and $V_{\mathrm{FG}}$ at room temperature as a function of applied magnetic field for $I_{\mathrm{AH}}=100 \mu \mathrm{A}$, as shown in Fig. 2a. As is evident from the experimental data, the different regions of the NW exhibits distinctly different Hall characteristics. For simplicity, let us first assume that the electrical dimensions are equal to the physical dimensions. This implicitly assumes that the size of the depletion region due to the $p$-n junction is electrically insignificant. An analytical approximation of the Hall voltage in the present geometry then allows for an intuitive understanding of the experimental data. Compensating for the hexagonal shell area and the finite contact size, the Hall voltage $V_{\mathrm{H}}$ can be approximately described by

$$
V_{H}=\frac{I_{\mathrm{AH}}}{q n A_{\mathrm{shell}} / w_{c}} B
$$

where $I_{\mathrm{AH}}$ is the current sourced through terminals $\mathrm{AH}, \mathrm{B}$ is the applied magnetic field, q is the elementary charge, $\mathrm{n}$ is the electron concentration, $A_{\text {shell }}$ is the hexagonal shell area and $w_{\mathrm{c}}$ is the distance between the points where the voltage terminals probe the potential. Using the present geometry, the linear fits shown in Figure 2a allow us to calculate the carrier concentration $n$. We find that the carrier concentration varies along the length of the NW and is calculated to be $n_{\mathrm{BC}}=3.3 \cdot 10^{18} \mathrm{~cm}^{-3}, n_{\mathrm{DE}}=9.9 \cdot 10^{17} \mathrm{~cm}^{-3}$ and $n_{\mathrm{FG}}=4.5 \cdot 10^{17} \mathrm{~cm}^{-3}$ between terminals $\mathrm{BC}$, DE and FG respectively. Already by using this simple analytical model, the NW Hall design has allowed us to estimate the spatially resolved carrier density in the shell. As is shown in the supplementary information Fig. 2, separate multi-probe resistivity measurements along the nanowire further corroborates these measurements by showing a position-dependent resistivity. The resistivity measurements always showed a transient going from high resistance at the NW tip, to low resistance at the NW base, consistent with a gradient in doping density. These findings are further supported by the rectifying properties of the current-terminals $\mathrm{A}$ and $\mathrm{H}$, where it is only possible to pass significant current in one direction, from the low doped end to the highly doped end, indicating that the Schottky contact on the low-doped end limits the current.

It is of course crucial to take into account any effects from the depletion region formed between the p-type core and n-type shell of the NW, and furthermore, to investigate the possibility that 
a gradient of the acceptor concentration in the core could potentially give rise to these results. In a previous work ${ }^{7}$, it was shown that the p-type core, grown under nearly identical conditions, exhibited a doping concentration of about $10^{18} \mathrm{~cm}^{-3}$. A core acceptor concentration gradient along the NW axis would give rise to a varying depletion width. This would in turn define the effective electrical thickness of the shell. However, an analysis of the experimental data, assuming that a varying shell depletion width is responsible for the difference in Hall signal, shows that the effect is too small to account for the order of magnitude change of the measured carrier density (Supplementary Information). We thus conclude that a core doping gradient alone cannot be responsible for the measured characteristics.

The doping concentrations and the effective electrical shell thickness is however coupled through the depletion width. In Figure 2b, we investigate the potential landscape in a cross-section of the nanowire using a 2D Poisson model. In the figure, the potential variation is plotted for a core acceptor concentration and shell donor concentration both equal to $10^{18} \mathrm{~cm}^{-3}$. Figure $2 \mathrm{c}$ shows the difference between the physical core radius, and the radius for which the electron concentration has dropped to $50 \%$ of its initial value, as a measure of the position of the boundary of the depletion region. For shell donor concentrations higher than $1 \cdot 10^{18} \mathrm{~cm}^{-3}$, it is seen that the depletion region only extends $\sim 10 \mathrm{~nm}$ into the NW shell. This amounts to a change in conduction area of less than $5 \%$. For this regime, this effect will thus be secondary, and a constant electrical shell thickness can be assumed. For donor concentrations less than $1 \cdot 10^{18} \mathrm{~cm}^{-3}$ however, this effect will be slightly larger, and should be taken into account for low concentrations. Similarly, it can be shown that variations in the core acceptor density an order of magnitude in either direction only introduces a small error in the evaluation of the experimental results. For simplicity, we will thus assume a doping-independent electrical shell thickness, except for the lowest donor concentrations, where a doping-dependent electrical shell thickness will be included in the analysis.

In order to understand the Hall effect in the hexagonal NW shell geometry beyond the simple approximation of Eqn. 1, the Hall effect was simulated in an identical 3D structure by solving the current continuity equation using the finite element method (implemented using COMSOL Multiphysics). The magnetic field was taken into account using the anisotropic conductivity tensor

$$
\sigma=\sigma_{0}\left(\begin{array}{ccc}
\frac{1}{1+(\omega \tau)^{2}} & -\frac{\omega \tau}{1+(\omega \tau)^{2}} & 0 \\
\frac{\omega \tau}{1+(\omega \tau)^{2}} & \frac{1}{1+(\omega \tau)^{2}} & 0 \\
0 & 0 & 1
\end{array}\right)
$$

where $\sigma_{0}$ is the isotropic conductivity, $\omega=\frac{q B}{m}$ is the cyclotron frequency with $\bar{B}=(0,0, B)$ the magnetic field, $\mathrm{m}$ the InP electron effective mass and $\tau$ the scattering time. See supplementary information, fig. 3 for more information.

Using the linear fits to the experimental results from Fig. 2a together with data generated from the simulations, accurate carrier densities are extracted. We find $n_{\mathrm{BC}}=5.6 \cdot 10^{18} \mathrm{~cm}^{-3}$, $n_{\mathrm{DE}}=1.7 \cdot 10^{18} \mathrm{~cm}^{-3}$ and $n_{\mathrm{FG}}=7.4 \cdot 10^{17} \mathrm{~cm}^{-3}$. These results show that the analytical model underestimates the carrier density slightly, but nevertheless gives a reasonable first approximation of the quantity of interest. It should be noted that depletion effects due to surface pinning of the Fermi energy was not taken into account in the simulations. These effects are expected to have only a minor impact on the measured carrier density in n-type $\mathrm{InP} \mathrm{NWs}^{16}$.

The resistivity can be determined by measuring the longitudinal potential drops $V_{\mathrm{BD}}, V_{\mathrm{DF}}, V_{\mathrm{CE}}$ and $V_{\mathrm{EG}}$. Using the measured carrier densities together with a Drude model, the mobility is then 
calculated to be $\mu_{B C}=198 \mathrm{~cm}^{2} / \mathrm{Vs}, \mu_{D E}=430 \mathrm{~cm}^{2} / \mathrm{Vs}$ and $\mu_{F G}=770 \mathrm{~cm}^{2} / \mathrm{Vs}$. These mobility values are consistent with previous InP nanowire mobility studies ${ }^{15}$, and are comparable with bulk InP values ${ }^{17}$. The NWs exhibit randomly distributed twin-plane stacking defects which affect the carrier mobility. It is plausible that the mobility may be increased by further optimizing the growth conditions.

At this point it is of interest to briefly discuss the range of applicability of the presented technology. For this letter, core-shell NWs intended for applications within LED and PV devices, and thus of quite large diameter, are characterized. It is however also important to investigate how small NWs that are compatible with the presented platform. Furthermore, the range of detectable carrier densities is of crucial interest. A detailed discussion is found in Figure 5 of Supplementary information, but work in progress indicates that NWs down to at least $80 \mathrm{~nm}$ is currently compatible, and in the future we expect to be able to characterize NWs down to $40 \mathrm{~nm}$ diameter. The Hall signal is reduced when the carrier density is increased, and is limited by the sensitivity of the measurement setup, as shown in fig. 5c of supplementary information.

A strong advantage of the presented measurement platform is the ability to combine multiple characterization techniques on the same, or a neighboring, NW. In order to independently verify the results from the Hall measurements, Cathodo-Luminescence (CL) measurements were performed locally along the length of two NWs on the same sample. By locally exciting the NWs using the electron beam, the luminescence from a small region of a NW can be investigated. The measurement setup is illustrated in figure 3 a.

A beam of electrons of energy $5 \mathrm{keV}$ was used to locally excite the material at different positions of the NW. The electron penetration in InP for the specified electron energy is estimated by MonteCarlo simulations (supplementary information, Fig. 4), implementing the Kanaya-Okayama ${ }^{18}$ model, to be $150 \mathrm{~nm}$, and the position for maximum energy transfer is shown to be approximately $44 \mathrm{~nm}$ below the surface of the NW, with $84 \%$ deposited in the NW shell. The beam will thus primarily excite the nanowire shell (of approximate thickness $120 \mathrm{~nm}$ ). Any excitation inside the depletion region of the p-n junction should not produce significant luminescence due to efficient separation of the generated carriers. We thus conclude that any measured luminescence will originate primarily from the shell of the core-shell p-n nanowire structure. By scanning the beam over different portions of the NW, local material properties of the shell can be probed and characterized. Fig. 3b shows three such spectra taken at three different positions along the length of a NW.

There is a clear shift in the luminescence peaks as we probe different regions along the NW, indicating a change of carrier density ${ }^{19}$. Following the procedure described by Bugajski et al. ${ }^{19}$, taking into account the bandgap shrinkage due to electron-electron and electron-impurity interaction, as well as the Burstein-Moss shift, the expected emission peak position as a function of carrier concentrations can be calculated, see Fig. 3c. Plotted in the figure is the calculated doping-dependent peak position (solid line) together with the experimentally determined luminescence peak positions fitted on top of the line for two different NWs. The only input parameter for the calculations is the zinc-blende temperature-dependent InP bandgap. The Varshni model ${ }^{20}$ was used to calculate the InP bandgap at $6.5 \mathrm{~K}$. As is evident from the figure, these measurements yield a doping concentration in the range of $5 \cdot 10^{17} \mathrm{~cm}^{-3}$ to $5 \cdot 10^{18} \mathrm{~cm}^{-3}$, in excellent agreement with the carrier densities extracted from the Hall measurements. The CL measurements thus provide a separate verification of the results obtained using the Hall measurements.

We have conclusively shown that the NWs chosen for this first demonstration of the technology exhibit a gradient in the carrier density of the n-type shell. This anomalous case, likely originating from variations in the effective V/III-ratio during growth, was chosen due to its excellent potential to 
demonstrate the spatially resolved single NW Hall measurements. More studies must be conducted to find the origin of this epitaxial behavior and further optimize the NWs for LED and PV device applications, and the presented technology constitutes a vital characterization platform for this purpose.

In summary, we demonstrate a novel platform for performing spatially resolved mapping of carrier density and mobility in NWs using the Hall effect. The presented platform furthermore enables multiple characterization technologies to be implemented on the same or neighboring NWs. We demonstrate the platform by characterizing the shell of core-shell InP nanowires intended for PV and IR LED applications. The measurements are simple, conclusive and are able to reveal a gradient in the shell doping profile along the NW. This method is expected to have significant impact and paves the way for future development of advanced engineered NW devices.

\section{Figure captions}

\section{Figure 1}

Device used to measure Hall effect on single nanowires. a, A current is fed through terminals A and $\mathrm{H}$ and the terminals $\mathrm{BC}, \mathrm{DE}$ and $\mathrm{FG}$ are used to locally probe the material properties using the Hall effect. All scalebars are $1 \mu \mathrm{m} \mathbf{b}$, The as-grown InP core-shell NWs, the scalebar is $1 \mu \mathrm{m}$. c, Details of the device design. The self-aligned lifting layer allows high spatial resolution of the contacts.

\section{Figure 2}

Experimental room temperature data. a, Hall data measured at room temperature at three different positions on the NW. The voltage offset due to the longitudinal voltage drop has been removed for clarity. Each datapoint was measured 100 times, and the errorbars show one standard deviation in each direction. $\mathbf{b}$, The potential distribution of the NW cross section for core and shell doping concentration of each $10^{18} \mathrm{~cm}^{-3}$, with the periphery of the NW taken to be at zero potential. The solid black line shows the physical boundary between the core and the shell. c, The shell depletion width as the donor concentration is varied. It is seen that a doping-dependent electrical shell thickness must be taken into account at low donor concentrations.

\section{Figure 3}

Cathodo-luminescence measurements. a, Illustration of the measurement setup. An SEM is used to locally generate electron-hole pairs. b, The CL spectra for three different positions on the NW. c, The solid line shows a calculation of the expected peak position as function of carrier density, and $\square$ and + shows the luminescence peaks for two different nanowires.

\section{Methods}

Gold catalysts were deposited on SiN-masked p-type InP (111)B substrates using nano imprint lithography and reactive ion etching. Growth took place in a low pressure (100 mbar) horizontal metal-organic chemical vapor deposition reactor. The core was grown using trimethyl-indium (TMIn), phosphine $\left(\mathrm{PH}_{3}\right)$, diethyl-zinc (DEZn) and $\mathrm{HCl}$ with molar fractions $\chi(\mathrm{TMIn})=8.2 \cdot 10^{-6}$, $\chi\left(\mathrm{PH}_{3}\right)=1.8 \cdot 10^{-2}, \chi(\mathrm{DEZn})=2.2 \cdot 10^{-6}$ and $\chi(\mathrm{HCl})=1.3 \cdot 10^{-5}$ respectively. The sample was 
subsequently removed from the growth chamber and the Au-particles were removed ex-situ in a $\mathrm{KI}: \mathrm{I}_{2}: \mathrm{H}_{2} \mathrm{O}$ solution. The sample was reinserted into the growth chamber and an n-type shell was grown using TMIn, $\mathrm{PH}_{3}$ and hydrogensulfide $\left(\mathrm{H}_{2} \mathrm{~S}\right)$ with molar fractions $\chi(\mathrm{TMIn})=1.8 \cdot 10^{-5}$, $\chi\left(\mathrm{PH}_{3}\right)=1.8 \cdot 10^{-2}$ and $\chi\left(\mathrm{H}_{2} \mathrm{~S}\right)=1.6 \cdot 10^{-6}$. The growth temperature was $395^{\circ} \mathrm{C}$ for the core and $550^{\circ} \mathrm{C}$ for the shell. The facet width of the hexagonal core was measured to be $86 \mathrm{~nm} \pm 4 \mathrm{~nm}$, and the shell facet width was measured to be $220 \mathrm{~nm} \pm 13 \mathrm{~nm}$.

Individual NWs were subsequently transferred to a $\mathrm{SiO}_{2}$-covered $\mathrm{Si}$ substrate with pre-defined bond pads. A lifting layer consisting of one part Shipley resist S1805 diluted with one part 2Methoxy-1-Methylethyl Acetate (PGMA) was spun onto the sample at $5000 \mathrm{rpm}$ and permanentbaked at $200^{\circ} \mathrm{C}$ for $10 \mathrm{~min}$. The surface of the thin diluted $\mathrm{S} 1805$ resist aligns itself to the highest point of the NWs and a window can easily be opened up using a short oxygen plasma process. This allows the process to be routinely applied to NWs of varying diameter without tuning the thickness of the lifting layer. This process is similar in concept to a previously published process using spin-on glass. $^{21}$

After a window to the NW was opened using an $\mathrm{O}_{2}$-plasma, contacts were exposed in PMMA using electron beam lithography. The surface was prepared by submersing the sample in buffered HF for 1 min followed by thermal evaporation of $5 \mathrm{~nm}$ and $100 \mathrm{~nm}$ of $\mathrm{Ti}$ and $\mathrm{Au}$ respectively. The liftoff was performed in acetone.

The Hall measurements were performed at room temperature and the CL measurements in a home-built CL system at $6.5 \mathrm{~K}$. A W-filament was used as thermionic emitter and a GaAs photomultiplier as detector. The spatial resolution was below $100 \mathrm{~nm}$ and the spectral resolution approximately $10 \mathrm{meV}$.

\section{References}

[1] Guo, W., Zhang, M., Banerjee, A. \& Bhattacharya, P. Catalyst-free InGaN/GaN nanowire light emitting diodes grown on (001) silicon by molecular beam epitaxy. Nano lett. 10, 33553359 (2010).

[2] Guo, W., Banerjee, A., Bhattacharya, P. \& Ooi, B.S. InGaN/GaN disk-in-nanowire white light emitting diodes on (001) silicon. APL 98, 193102 (2011).

[3] Hersee, S. et al. GaN nanowire light emitting diodes based on templated and scalable nanowire growth. Electron. Lett. 45, 75-76 (2009).

[4] Kuykendall, T., Ulrich, P., Aloni, S. \& Yang, P. Complete composition tunability of InGaN nanowires using a combinatorial approach. Nature mater. 6, 951-956 (2007).

[5] Qian, F., Gradecak, S., Li, Y., Wen, C.-Y. \& Lieber, C.M. Core/multishell nanowire heterostructures as multicolor, high-efficiency light-emitting diodes. Nano lett. 5, 2287-2291 (2005).

[6] Svensson, C.P.T. et al. Monolithic GaAs/InGaP nanowire light emitting diodes on silicon. Nanotechnology 19, 305201 (2008).

[7] Borgström, M., Wallentin, J. \& Heurlin, M. Nanowires with promise for photovoltaics. IEEE J. Sel. Top. Quant. 17, 1050-1061 (2010). 
[8] Czaban, J.A., Thompson, D.A. \& LaPierre, R.R. GaAs core-shell nanowires for photovoltaic applications. Nano lett. 9, 148-154 (2009).

[9] Kayes, B.M., Atwater, H.A. \& Lewis, N. S. Comparison of the device physics principles of planar and radial p-n junction nanorod solar cells. JAP 97, 114302 (2005).

[10] Kelzenberg, M.D. et al. Photovoltaic measurements in single-nanowire silicon solar cells. Nano lett. 8, 710-714 (2008).

[11] Tsao, J. Solid-state lighting: lamps, chips, and materials for tomorrow. IEEE Circuits Devices Mag. 20, 28-37 (2004).

[12] Connors, B., Povolotskyi, M., Hicks, R. \& Klein, B. Simulation and design of core-shell GaN nanowire LEDs. Simulation 7597, 75970B (2010).

[13] Roddaro, S. et al. InAs nanowire metal-oxide-semiconductor capacitors. APL 92, 253509 (2008).

[14] Tu, R., Zhang, L., Nishi, Y. \& Dai, H. Measuring the capacitance of individual semiconductor nanowires for carrier mobility assessment. Nano lett. 7, 1561-1565 (2007).

[15] Borgström, M.T. et al. Precursor evaluation for in situ InP nanowire doping. Nanotechnology 19, 445602 (2008).

[16] Aspnes, D. Recombination at semiconductor surfaces and interfaces. Surf. Sci. 132, 406-421 (1983).

[17] Anderson, D.A., Apsley, N., Davies, P. \& Giles, P.L. Compensation in heavily doped n-type InP and GaAs. JAP 58, 3059 (1985).

[18] Kanaya, K. Penetration and energy-loss theory of electrons in solid targets. J. Phys. D: Appl. Phys. 5,43-58 (1972).

[19] Bugajski, M. \& Lewandowski, W. Concentration-dependent absorption and photoluminescence of n-type InP. JAP 57, 521 (1985).

[20] Varshni, Y. Temperature dependence of the energy gap in semiconductors. Physica 34, 149-154 (1967).

[21] Zimmler, M. A et al. Scalable fabrication of nanowire photonic and electronic circuits using spin-on glass. Nano lett. 8, 1695-1699 (2008).

\section{Acknowledgments}

This project is performed within the Nanometer Structure Consortium at Lund University (nmC@LU) and with financial support from the Swedish Research Council (VR), the Swedish Foundation for Strategic Research (SSF), the Knut and Alice Wallenberg Foundation (KAW), VINNOVA, E.ON AG and the Nordic Innovation program NANORDSUN. We thank Ying-Lan Chang, Dan Csontos and Magnus Borgström for discussions. 


\section{Author contributions}

L. S. Designed the experiments and supervised the project. K. S. helped design the experiment, carried out simulations, took part in all measurements and wrote the paper. F. H. helped design and fabricated the Hall devices and performed the Hall and resistivity measurements. M. H. developed and grew the epitaxial nanowires. D. L. and A. G. performed the CL measurements. P. W. and B. M. took part in data interpretation and discussions.

\section{Additional information}

The authors declare no competing financial interest. Supplementary information accompanies this paper at www.nature.com/naturenanotechnology. Reprints and permission information is available online at http://www.nature.com/reprints. Correspondence and requests for materials should be addressed to L.S. 